Communications in Physics, Vol.21, No. 2 (2011), pp. 125-129

\title{
TWO BAND MODEL FOR DILUTED MAGNETIC SEMICONDUCTORS: STUDY OF THE FERROMAGNETIC TRANSITION TEMPERATURE
}

\author{
VU KIM THAI AND HOANG ANH TUAN \\ Institute of Physics, VAST
}

\begin{abstract}
The ferromagnetic transition temperature $\left(T_{c}\right)$ of a two band model for diluted magnetic semiconductors (DMS) is calculated by using the coherent potential approximation (CPA). It is shown that $T_{c}$ is strongly parameter dependent on density of the carriers, magnetic coupling constants, and the hopping terms. The maximal $T_{c}$ of the two band model is found when both impurity bands fully overlap and this value is approximately twice larger than the highest $T_{c}$ obtained in the single band model.
\end{abstract}

\section{INTRODUCTION}

The discovery of ferromagnetic order induced by doping common III-V semiconductors has recently generated a great deal of interest, because DMS are candidates for new functional materials whose magnetic properties are controllable by changing the carrier density. These new materials are believed to be a fundamental element to establish a spin-based electronics (spintronics) as a practical technology. From the application point of view, the low Curie temperature of the investigated DMS represent a serious problem, and many efforts have been devoted to find DMS with $T_{c}$ higher than room temperature. To predict DMS with high $T_{c}$ is still a challenging task for theory, because the underlying mechanism of their ferromagnetism has not been completely understood. For the theoretical modeling of DMS mainly two basic approaches are used: ab initio or first principles studies and theories starting from effective Hamiltonians containing experimentally determined parameters. One class of models neglects interband coupling effects and only investigates the disorder of the localized moments [1-2]. Some models introduce the interband coupling between the itinerant carriers and the localized moment and study the effect of charge carriers on the magnetic phase diagram [3-4]. If the ferromagnetic order is mediated by "free" carriers, its origin is assumed to be Ruderman-Kittel-Kasuya-Yosida (RYYK) type [5]. The RKKY approach within mean field and continuous approximations is commonly referred as Zener mean field theory. In the Zener mean field theory, the Curie temperature for a fixed concentration of impurities varies with simple power law $\left(T_{c} \sim n^{1 / 3}\right)$ of the density of itinerant carriers [6]. However, further results show that RKKY interaction between localized spins is insufficient [7-8]. The impurity band model, where $p$ holes move around interacting with localized spins at Mn sites through the antiferromagnetic exchange interaction, is a widely used model for (III,Mn)V-type DMS . Predictions about Curie temperature in the one band model for DMS are made using 
Monte Carlo (MC) technique, dynamical mean field theory (DMFT), coherent potential approximation method and standard mean field approach [9-12]. It has been found that $T_{c}$ is maximized for the carrier density roughly half of the concentration of localized spins. In addition, for over-doping of the carriers, $T_{c}$ decreases to zero.

Recently the critical temperature was calculated using a two band model for DMS by MC technique and DMFT [13]. The new finding is that the maximal $T_{c}$ in the two band model at carrier filling $n \approx x$ is approximately twice larger than the highest $T_{c}$ obtained in the single band model at filling $n \approx x / 2$, where $x$ is Mn density. The purpose of this paper is to calculate the Curie temperature of the two band model for DMS and study its dependence on the system parameters by using CPA. As the results obtained within DMFT and Monte Carlo simulation approach, our results show the importance of multiband descriptions of DMS.

\section{THE MODEL AND FORMALISM}

It is well known that in Mn-doped GaAs, the Mn ions substitute for Ga cations and contribute holes to the valence band and the effective spin of holes is $3 / 2$ [5]. Because of spin-orbit interaction, the p-orbitals are split into a spin- $3 / 2$ multiplet and a spin- $1 / 2$ multiplet. This is the reason why we choose to study two bands since this is the relevant number of orbitals in most III-V DMS. The simple two band model for DMS used here is given by

$$
H=\sum_{l i j \sigma} t_{l}\left(c_{l i \sigma}^{+} c_{l j \sigma}+\text { H.c. }\right)+\sum_{l, I} J_{l} \mathbf{S}_{I} \mathbf{s}_{l i},
$$

where $l=1,2$ is the band index, $t_{l}$ is the hopping term in the band $l, c_{l i \sigma}^{+}\left(c_{l j \sigma}\right)$ is the creation (annihilation) operator for a carrier at site $i$ with spin $\sigma$ in the band $l$, $\mathbf{s}_{l i}=c_{l i \alpha}^{+}\left(\frac{1}{2} \sigma_{\alpha \beta}\right) c_{l i \beta}$ is the spin operator at site $i$ of itinerant electron gas, $J_{l}$ is the coupling between the core spin and electrons of band $l$, and $\mathbf{S}_{I}$ is the spin of localized ion at randomly selected sites $I$. Here we treat the localized spins classically $(S=1 / 2)$, though the extension to the case $S=5 / 2$ is straightforward [14-15].

We apply CPA $[9,16]$ to the above model. In CPA the carriers are described as independent particles moving in an effective medium of spin-dependent coherent potentials. The coherent potential $\Sigma_{l, \sigma}(\sigma=\uparrow, \downarrow)$ is determined by demanding the scattering matrix for a carrier at an arbitrarily chosen site embedded in the effective medium vanished on average. By using a bare semicircular noninteracting density of states (DOS) with halfbandwidth

$W_{l}$ for the band $l: \rho_{l 0}(z)=\frac{2}{\pi W_{l}^{2}} \sqrt{W_{l}^{2}-z^{2}}$ we obtain the following equation for the Green function for each band for a given magnetization $m$

$$
G_{l \sigma}(\omega)=\frac{1-x}{\omega-w_{l} G_{l \sigma}(\omega)}+\frac{x(1+m) / 2}{\omega-w_{l} G_{l \sigma}(\omega)+J_{l} \sigma}+\frac{x(1-m) / 2}{\omega-w_{l} G_{l \sigma}(\omega)-J_{l} \sigma},
$$

where $w_{l}=W_{l}^{2} / 4$ and $\sigma= \pm 1$.

The Eq. (2) is easily transformed into a quartic equation for $G_{l \sigma}(\omega)$ and it is solved analytically by using Farrari method. The total DOS per site is then given by $\rho_{\text {tot }}(\omega)=$ $\sum_{l \sigma} \rho_{l \sigma}(\omega)$, where $\rho_{l \sigma}(\omega)=-1 / \pi \Im G_{l \sigma}(\omega)$. Throughout this work, we assume that the 
carriers are degenerate. Then the carrier density and the total energy can be expressed as

$$
\begin{array}{r}
n=\int_{-\infty}^{\mu} \rho_{t o t}(\omega) d \omega, \\
E(m)=\int_{-\infty}^{\mu} \omega \rho_{t o t}(\omega) d \omega,
\end{array}
$$

where $\mu$ is the chemical potential. The free energy per site of the system at temperature $T$ is given as

$$
F(m)=E(m)-T S,
$$

where the entropy due to the localized spins is given by

$$
\mathcal{S}=x k_{B} \ln \sum_{S^{z}=-S}^{S} \exp \left(\frac{h S^{z}}{k_{B} T}\right)-\frac{h m}{T} x .
$$

Here $h$ denotes the effective field felt by the localized spins. By minimizing $F$ with respect to $m$ we obtain the following equation for $h$

$$
h=-\frac{1}{x} \frac{d E(m)}{d m} .
$$

The local magnetization is then calculated by

$$
m=S B_{S}\left(\frac{h S}{k_{B} T}\right),
$$

where $B_{S}(x)$ is the conventional Brillouin function.

The Curie temperature is determined by differentiating both sides of Eq. (8) with respect to $m$ at $m=0$. This leads to the formula

$$
k_{B} T_{c}=\left.\frac{S(S+1)}{3} \frac{d h}{d m}\right|_{m=0} .
$$

By setting $J_{2}=0$ we reproduce the one band result for $T_{c}$ of Ref. 9 .

\section{NUMERICAL RESULTS AND DISCUSSION}

In this work we take $W_{1}$ as the unit of energy. We focus on the doping of $x=0.05$ associated with the highest $T_{c}$ in $\mathrm{Ga}_{1-x} \mathrm{Mn}{ }_{x}$ As. The results for the Curie temperature as a function of carrier density $n$ are plotted in Figs. 1-3. We want to compare our results with the ones in Ref. 13, so we have calculated $T_{c}(n)$ varying exchange couplings, bandwidths and the exchange-to-bandwidth ratio as well. Fig. 1 displays $T_{c}(n)$ for $W_{1}=W_{2}=J_{1}$ and different values of $J_{2} / J_{1}$. Our resulting curves are very similar to the ones in Ref. 13: at all $n T_{c}$ is maximum when the impurity band (IB) fully overlap $\left(J_{1}=J_{2}\right)$; the curves with $J_{2} / J_{1}=1.15$ and 1.30 correspond to partially overlapping bands, while that with $J_{2} / J_{1}=0.55$ corresponds to decoupled IB. In Fig. 2 we present $T_{c}$ vs $n$ at fixed $J_{1}=J_{2}=W_{1}$ for varying bandwidths $W_{2} / W_{1}$. Again, the curves strongly depend on the degree of overlapping of the IB and $T_{c}$ is maximal for all $n$ when the bands fully overlap $\left(W_{2} / W_{1}=1\right)$. The main difference between our curves and the ones obtained by DMFT in 


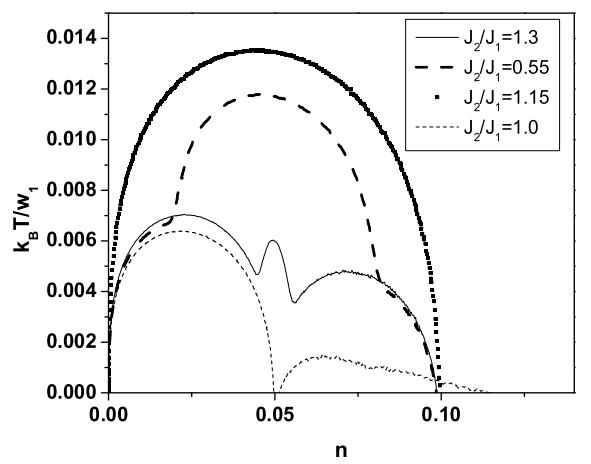

Fig. 1. Curie temperature as a function of carrier density for various $J_{2} / J_{1}$ for $x=0.05$ and $W_{1}=W_{2}=J_{1}$.

Ref. 13 is that our $T_{c}(n)$ is always nonzero in the range from $n=0$ to $n \approx x$ independent on the location of the IB. As the degree of overlapping of IB increases, a broad peak at $n \approx x$ is formed and the form of $T_{c}(n)$ curve becomes approximately semi-elliptical when the overlap is maximal $\left(W_{1}=W_{2}\right)$.

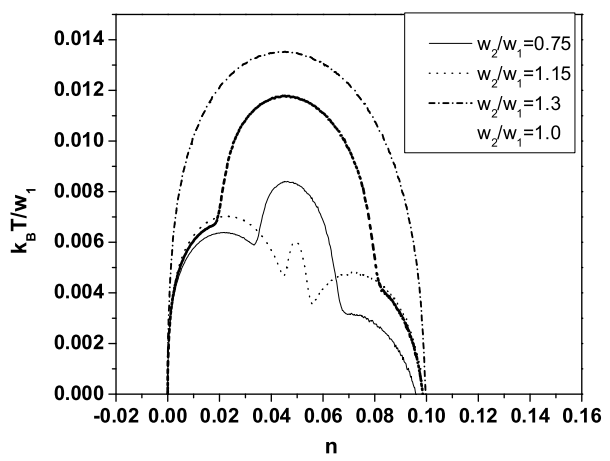

Fig. 2. Curie temperature as a function of carrier density for various $W_{2} / W_{1}$ for $x=0.05$ and $J_{1}=J_{2}=W_{1}$.

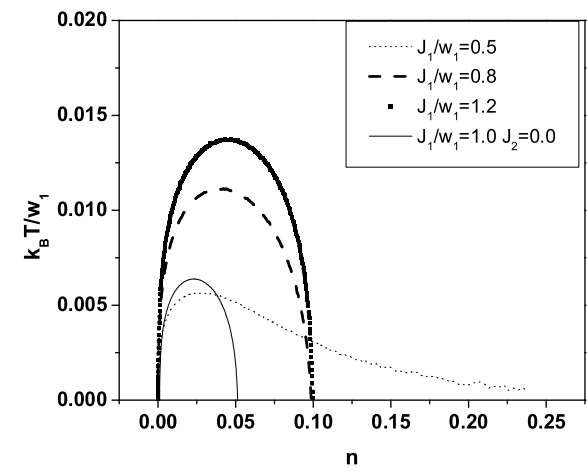

Fig. 3. Curie temperature as a function of carrier density for various $J_{1} / W_{1}$ for $x=$ $0.05, W_{1}=W_{2}$ and $J_{1}=J_{2}$.

Fig. 3 shows $T_{c}(n)$ when the IB fully overlap $\left(J_{1}=J_{2}, W_{1}=W_{2}\right)$ varying exchangeto-bandwidth ratio $J / W$. Our results are qualitatively and quantitatively comparable to those of Ref. 13: At small coupling $T_{c}$ is small and plat; at intermediate coupling $(0.8 \leq J / W \leq 1.2) T_{c}$ is nonzero in the range from $n=0$ to $n=2 x$ with the maximum at $n \approx x$. Additionally, $T_{c}$ at $n \approx x$ in the two band model approximately is two times larger than $T_{c}$ at filling $n \approx x / 2$ in the one band model. As was noted in Ref. 13, the explanation is straightforward: at $n=x$ in the one band model the IB is fully occupied leading to a vanishing $T_{c}$, but for the same $n$ in the two band model both bands are half 
filled, which ultimately leads to the highest value for $T_{c}$. The excellent agreement between our CPA results and those by DMFT is not surprising due to the similar approximations being used in both methods, in particulary, both CPA and DMFT are good approximation when the dimensionality goes to infinity.

To conclude, we summarize our results here. In this paper the coherent potential approximation has been naturally extended to a two-band model for DMS. The Curie temperature has been calculated by minimizing the free energy of the coupled carrier-localized spin systems. It has been predicted that Curie temperature $T_{c}$ is maximized at all carrier densities when both impurity bands fully overlap. In this regime $T_{c}$ is increased as the carrier density increases from to $n=0$ to $n \approx x$ and the maximal $T_{c}$ of the two band model is approximately twice larger than the highest $T_{c}$ obtained in the single band model. Our results for $T_{c}(n)$ are in good agreement with the ones obtained by DMFT and MC technique. In particulary, it supports the view that semiconductors with the smallest heavy-to-light hole mass ratio could have highest $T_{c}$ if the magnetic couplings are optimized. In this work, we focus only on multi-band effect of DMS. The complexity of the problem did not yet allow us to include the inspection of the band anisotropy, the nonmagnetic potential, the direct antiferromagnetic exchange interaction between Mn ions which is intended for the near future.

\section{ACKNOWLEGMENTS}

This work is supported by the National Foundation for Science and Technology Development (NAFOSTED).

\section{REFERENCES}

[1] C. Zhou et al., Phys. Rev., B69 (2004) 144419.

[2] G. X. Tang and W. Nolting, Phys. Rev., B73 (2006) 024415.

[3] M. Berciu and R. N. Bhatt, Phys. Rev. Lett., 87 (2001) 107203.

[4] G. Bouzerar, J. Kudrnovsky, and P. Bruno, Phys. Rev., B68 (2003) 20531.

[5] G. Zarand and B. Janko, Phys. Rev. Lett., 89 (2002) 047201.

[6] T. Dietl, H. Ohno and F. Matsukura, Phys. Rev., B63 (2001) 195205.

[7] G. A. Fiete at al., Phys. Rev., B71 (2005) 115202.

[8] R. Bouzerar et al. Phys. Rev., B73,(2006) 024411.

[9] M. Yagi and Y. Kayanuma, J. Phys. Soc. Jpn., 71 (2002) 2010.

[10] K. Hirakawa et al., Phys. Rev., B65 (2002) 193312.

[11] T. T. Jungwirth et al., Phys. Rev., B59 (1999) 9818.

[12] M. Takahashi and K. Kubo, J. Phys. Soc. Jpn., 72 (2003) 2866.

[13] F. Popescu et al., Phys. Rev., B73 (2006) 075206.

[14] S. S. Feng and M. Mochena, J. Phys.: Condens. Matter, 18 (2006) 1441.

[15] Anh-Tuan Hoang Physica B, 403 (2008) 1803.

[16] Hoang Anh Tuan and Le Duc Anh, Commun. Phys., 15 (2005) 199.

Received 30 October 2010. 